\title{
Microbial biodiversity assessment of the European Space Agency's ExoMars 2016 mission
}

Kaisa Koskinen ${ }^{1,2}$, Petra Rettberg ${ }^{3 *}$, Rüdiger Pukall ${ }^{4}$, Anna Auerbach $^{5}$, Lisa Wink ${ }^{1}$, Simon Barczyk ${ }^{3}$, Alexandra Perras ${ }^{1,5}$, Alexander Mahnert ${ }^{6}$, Diana Margheritis ${ }^{7}$, Gerhard Kminek ${ }^{8}$ and Christine Moissl-Eichinger ${ }^{1,2^{*}}$

\begin{abstract}
Background: The ExoMars 2016 mission, consisting of the Trace Gas Orbiter and the Schiaparelli lander, was launched on March 142016 from Baikonur, Kazakhstan and reached its destination in October 2016. The Schiaparelli lander was subject to strict requirements for microbial cleanliness according to the obligatory planetary protection policy. To reach the required cleanliness, the ExoMars 2016 flight hardware was assembled in a newly built, biocontrolled cleanroom complex at Thales Alenia Space in Turin, Italy. In this study, we performed microbiological surveys of the cleanroom facilities and the spacecraft hardware before and during the assembly, integration and testing (AIT) activities.
\end{abstract}

Methods: Besides the European Space Agency (ESA) standard bioburden assay, that served as a proxy for the microbiological contamination in general, we performed various alternative cultivation assays and utilised molecular techniques, including quantitative PCR and next generation sequencing, to assess the absolute and relative abundance and broadest diversity of microorganisms and their signatures in the cleanroom and on the spacecraft hardware.

Results: Our results show that the bioburden, detected microbial contamination and microbial diversity decreased continuously after the cleanroom was decontaminated with more effective cleaning agents and during the ongoing AIT. The studied cleanrooms and change room were occupied by very distinct microbial communities: Overall, the change room harboured a higher number and diversity of microorganisms, including Propionibacterium, which was found to be significantly increased in the change room. In particular, the so called alternative cultivation assays proved important in detecting a broader cultivable diversity than covered by the standard bioburden assay and thus completed the picture on the cleanroom microbiota.

Conclusion: During the whole project, the bioburden stayed at acceptable level and did not raise any concern for the ExoMars 2016 mission. The cleanroom complex at Thales Alenia Space in Turin is an excellent example of how efficient microbiological control is performed.

Keywords: ExoMars, Planetary protection, Life-detection, Astrobiology, Cleanroom microbiota

\footnotetext{
* Correspondence: Petra.Rettberg@dlr.de; christine.moissl-

eichinger@medunigraz.at

${ }^{3}$ Radiation Biology Department, German Aerospace Center (DLR), Institute of

Aerospace Medicine, Cologne, Germany

${ }^{1}$ Department for Internal Medicine, Section of Infectious Diseases and

Tropical Medicine, Medical University of Graz, Graz, Austria

Full list of author information is available at the end of the article
} 


\section{Background}

Finding life outside the terrestrial biosphere is one of the drivers for humans to venture beyond Earth. The reports on numerous habitable planets have fuelled the speculations and hopes for the existence of extra-terrestrial life ([1]; NASA Exoplanet Archive; http://exoplanetarchive.ipac.caltech.edu/). However, these potentially life-bearing planets are far beyond human's reach-except Mars, where the special regions provide conditions in terms of temperature and water activity that would allow the propagation of terrestrial life on Mars today [2, 3].

No Martian life forms are yet known, but the observation of gaseous methane outbreaks in the Martian atmosphere has raised tremendous interest. Methane was frequently detected in the thin Martian atmosphere by the Planetary Fourier Spectrometer on ESA's Mars Express, or other Earth- and Mars-based instruments $[4,5]$. On Earth, methane is an excellent signature for microbial activity: More than $90 \%$ of the methane detected on Earth has been produced by microorganisms [6]. On Mars, the origin of methane is still subject of speculation. Potential sources are production in magma, the serpentinization of basalt (olivine, pyroxene) - or the activity of methanogenic microorganisms in a permafrost-like setting [6]. However, methane can remain archived in frozen reservoirs in form of methane clathrate for long periods of time, so if the origin is biogenic, the time point of potential microbial methane production remains unclear.

To date, numerous missions have been sent to Mars during the last decades to find life, signatures thereof or suitable environmental conditions for microbial life. NASA's Viking program (1975), the Mars Exploration Rover Mission (2003), the Phoenix lander (2008), and the Mars Science Laboratory including Curiosity rover (2012) were still not able to return clear positive results. However, with the ability to explore extra-terrestrial environments directly with spacecraft there comes great responsibility: contamination of the instruments and potential extra-terrestrial ecosystem by accidentally transferred terrestrial microorganisms could tremendously affect the actual, but also future scientific missions and the planetary body itself.

In order to limit the microbial contamination via space missions, obligatory rules for all spacefaring nations have been put in place half a century ago (Planetary Protection Policy, maintained by the Committee on Space Research (COSPAR), in line with Article IX of the United Nations Outer Space Treaty from 1967; [3]). These rules regulate the mission's category, depending on its target, type and purpose, and limit the acceptable microbial contamination level accordingly [3]. However, Earth is a microbial world, and even the human body carries $4 \times 10^{13}$ microbial cells [7] that are constantly spread into our environment [8]. As a consequence, control of microbial contamination results in a substantial effort, and needs to be implemented from the beginning of a mission planning. The number and diversity of microorganisms in close vicinity of a spacecraft during AIT (assembly, integration and test) activities are dependent on a variety of factors, such as cleanroom class and architecture, the clothing and behaviour of personnel, and the cleaning and disinfection protocol of the facility [9-11].

Modern spacecraft carry extremely sensitive equipment and instruments, and therefore cannot be easily sterilised as a whole after assembly. Consequently, spacecraft parts are often sterilised before integration, and integration is performed in bioburden controlled cleanrooms, where the spacecraft is subjected to a thorough microbial contamination control. Throughout the process, the level of microbiological contamination (bioburden) is examined carefully, and detected microorganisms are catalogued.

The bioburden control of spacecraft hardware was implemented already for the Viking mission [12]. At that time, bacterial spores were considered the hardiest forms of life on Earth, and cultivation-based procedure was developed for the assessment of the bioburden of a spacecraft, aiming to detect heat-shock resistant bacterial spores [12]. These bioburden-detection standard protocols are still used by the space agencies as a standard proxy for microbial cleanliness of a spacecraft $[13,14]$, but are nowadays complemented by molecular, NGS-based microbial community analysis to fully assess the associated microbiota [15-18]. In addition, the ESA standard has undergone improvements such as the implementation of the Millipore Milliflex system, allowing more rapid bioburden assessment, and the validation of more efficient sampling tools [19].

The awareness that spacecraft associated microorganisms need to be investigated and catalogued in order to improve microbial detection and sterilisation for future missions has initiated numerous studies on cleanroom and spacecraft microbiology (e.g. [20, 21]). Although the largest fraction of microorganisms detectable in cleanrooms seems to be dead or present as spores [22, 23], the diversity of survival specialists is considerable. Particularly, human-associated microorganisms, including bacteria, archaea, fungi and viruses are frequently detected [10, 22, 24]. ESA and NASA have implemented public culture collections, enabling the distribution of microbial isolates, with the goal to learn as much as possible on the resistance strategies of these microbes, and how to control these amazing survivors [25, 26].

ExoMars is a two-stage program of the European Space Agency (ESA) in cooperation with Roskosmos and contributions from NASA with the goal to detect signatures of extinct or present life on Mars. The first mission of the program arrived at Mars in October 2016 and consisted of two parts: the Trace Gas Orbiter (TGO) 
and an entry, descent and landing demonstrator module (Schiaparelli). The instruments were intended to study the water and geochemical setting, and measure and map methane. The second part of the program is a lander and a rover with the Pasteur payload and a 2-m drill [3], to be launched in 2020.

Following the planetary protection requirements, risks of crashing and biocontamination have to stay under strict limits. For TGO the chance of crashing on Mars has to be less than 1 in 100 for the first 20 years after launch. For the Schiaparelli lander strict biological contamination constraints were applicable. To meet these constraints the ExoMars project built a new microbiologically controlled cleanroom (BCCCR) and microbiology laboratory for Schiaparelli at Thales Alenia Space, Turin, Italy. This article reports the results from four sampling campaigns in this BCCCR, before and during the Schiaparelli spacecraft was prepared for launch.

\section{Methods}

\section{Cleanroom characteristics}

Throughout the time frame of three years, the cleanroom complex at Thales Alenia Space in Turin, Italy, was microbiologically sampled, before the different components of the Schiaparelli hardware were moved into the cleanroom, and during the time the assembly and integration activities were performed on the Schiaparelli hardware. The microbiologically controlled cleanroom complex in Turin has been built and is maintained under the responsibility of Thales Alenia Space, Italy, in the frame of the ExoMars program. The complex is built up of three cleanrooms and one change room, where the personnel don the cleanroom garments before entering the cleanroom. The change room (room 03) is connected via air-shower to cleanroom $4 \mathrm{~b}$ and $4 \mathrm{a}$, which is separated from the cleanroom 02 with a roll-up door (Fig. 1). The cleanroom 02 is a transfer room for all equipment, and place where the cleaning appliances are stored. The change room and cleanrooms $4 \mathrm{~b}$ and $4 \mathrm{a}$ are classified as microbiologically controlled ISO 7, and cleanroom 02 as microbiologically controlled ISO 8 (Fig. $1)$. The cleanroom classification is based on $0.5-\mu \mathrm{m}$ sized and larger particles with limits at $3.52 \times 10^{5}$ for ISO 7 per $\mathrm{m}^{3}$ air (ISO standard for cleanrooms and associated controlled environments; https://www.iso.org/ obp/ui/\#iso:std:iso:14644:-1:ed-1:v1:en). The cleanliness class is reached with a suitable number of air exchanges per hour, use of HEPA (high efficiency particulate air filter) filter unit, and a rigorous cleaning routine and strict access control.

During the entire time frame, the cleanroom was cleaned daily by dusting the floor, tables, shelves, doors, handles, and walls using sterile cloths. The cleaning agent used for decontamination varied during the assembly, integration and testing period (Additional file 1: Table S1). During each cleaning cycle, the cleaning started from the cleanest area (ISO $7 \mathrm{HC}$ ), and continued towards the less clean change room (03). The flight hardware was covered during cleaning. All the cleaning support tools (cart, brooms and buckets) were cleaned with IPA/sporicide, and kept in the ISO 8 room. The personnel underwent medical checks and specific trainings, in order to keep the microbial contamination as low as possible.

Overall, the flight hardware underwent numerous cleaning and sterilisation cycles, including application of $70 \%$ isopropyl alcohol cleaning and dry-heat bioburden reduction. More than 3000 routine microbiological (bioburden) tests of spacecraft and cleanroom surfaces and air were performed during the assembly of Schiaparelli. Personnel was specifically trained and had to wear full body garments, including face masks and sterilised gloves, and was medically checked with respect to infections and skin diseases, and the access of people was normally restricted to four to five. During testing at Thales Alenia Space in Cannes, France, a portable "clean tent" was installed in a ISO 8 cleanroom to guarantee the microbial integrity of the spacecraft. Before launch at Baikonur on March 14, 2016, the Schiaparelli spacecraft had to be transported from Italy to Kazakhstan. The entire transport required numerous logistical precautions, including double-sealing, specific air filters and a specific design of a container. In the cleanroom close to the launch site, the spacecraft was again protected from contamination using the "clean tent" for conducting further assembly steps and testing of the Schiaparelli lander.

\section{Sampling specifics, tools and locations}

During the period of interest, the cleanroom complex was sampled four times, namely in September 182013 (sampling campaign 06), May 272014 (08), December 8 2014 (11) and February 182015 (12). The first sampling took place before the flight hardware were brought in for integration immediately after commissioning. All procedures are described in detail in the ECSS (European Cooperation for Space Standardisation) document ECSS-Q-ST-70-55C [13]. Samples were collected from floor (areas approx. $1 \mathrm{~m}^{2}$ ), walls and facility structures, ground support equipment (GSE) and spacecraft hardware by using gamma sterilised bonded polyester cleanroom wipes (TX3211; ITW Texwipe, Kernesville, NC, USA). Wipes for bioburden and alternative cultivation assays were, before sampling, pre-moistened with $15 \mathrm{ml}$ water and autoclaved, and for cultivation of anaerobes, wipes were autoclaved dry. Wipes to be used for molecular analyses were first baked at $170{ }^{\circ} \mathrm{C}$ for $24 \mathrm{~h}$ (to destroy remnants of DNA), then pre-moistened with 


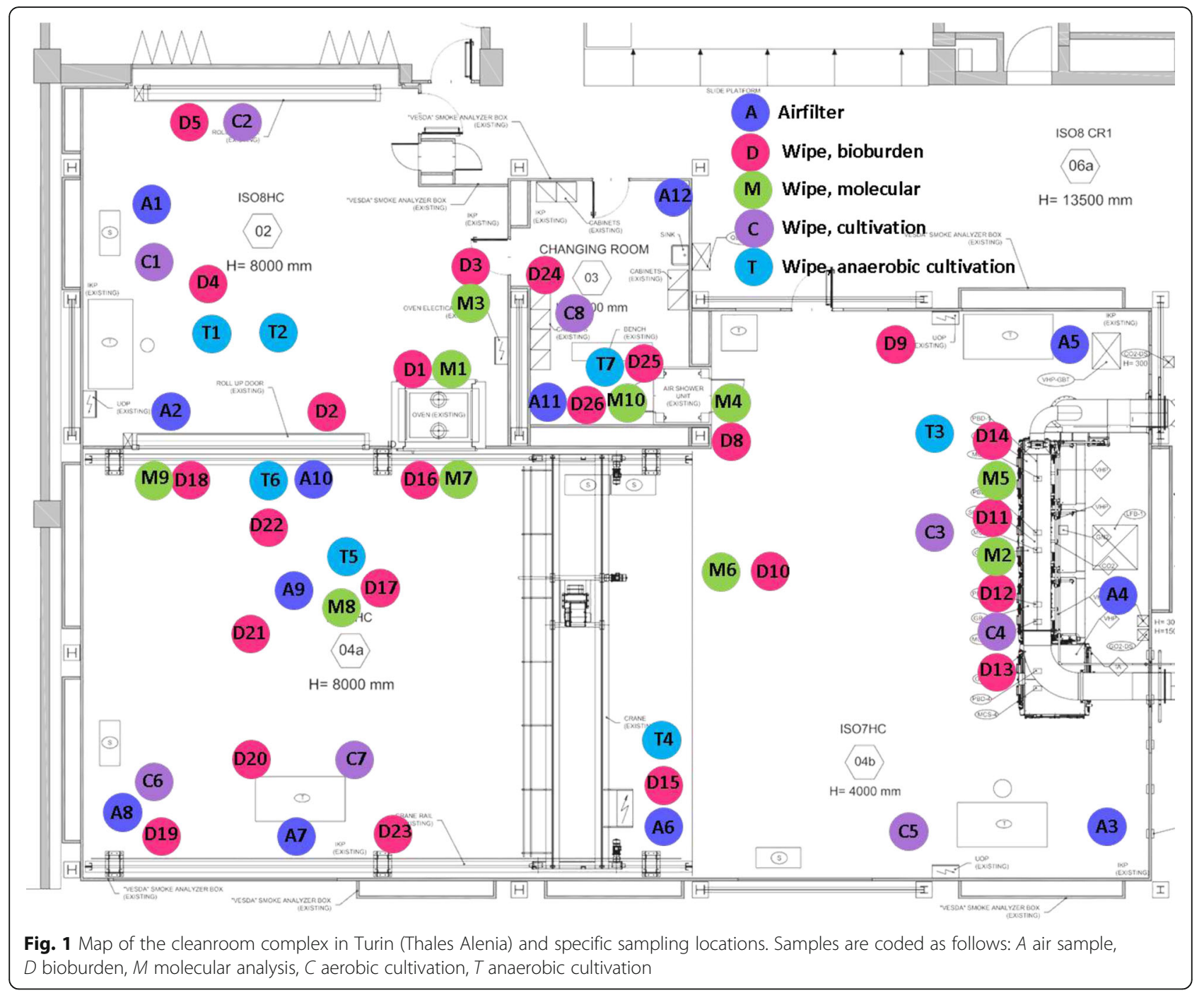

$15 \mathrm{ml}$ sterile water (microfiltered at $0.2 \mu \mathrm{m}$; LiChrosolv, Merck Millipore), and finally autoclaved in sterile and pyrogen-free PP tubes (Sarstedt, Germany). Smaller parts of the spacecraft hardware were sampled with nylon-flocked swabs (Copan FLOQSwab 552C, COPAN, Italy). The sampling gloves for aerobic and anaerobic cultivation samples were sterilised by autoclaving, and the DNA free gloves for molecular sampling were first UV-sterilised and then autoclaved. Samples were collected by using always a new, sterile and DNA free pair of gloves for each new wipe sample. For wipe sampling, the wipe was placed flat on the surface and rubbed over the entire surface using a firm, steady pressure. The same sample area was wiped a total of three times, rotating the direction of motion first $90^{\circ}$ and then $135^{\circ}$. The field negative control samples were collected by removing a sterile sampling wipe from its sterile and pyrogenfree PP tube, opening the wipe and placing it back to the tube. In laboratory, extraction blank samples were used to control the sterility of reagents and equipment. Controls were processed and analysed in the same way as samples.

Air samples were taken using a Sartorius AirPortMD8 device. Five hundred litres of air were sampled with a flow rate of $30 \mathrm{~L} / \mathrm{min}$, and the samples were collected on disposable gelatine filters [25]. Except for the spacecraft hardware the same sampling spots were sampled in each of the sampling campaigns. The sampling locations are given in Fig. 1 and described in more detail in Additional file 2: Table S2, Additional file 3: Table S3, Additional file 4: Table S4 and Additional file 5: Table S5.

Samples were taken for i) bioburden measurements (aiming at heat-shock resistant microorganisms, mostly spores), ii) biodiversity measurements ("alternative assays", aiming at oligotrophic, alkaliphilic, mesophilic and anaerobic microorganisms as well as fungi) and iii) molecular measurements (16S rRNA gene sequencingbased microbial community analyses). For each of the 
procedures, different samples were taken and processed independently, including field blanks and laboratory extraction controls. All samples were transported to laboratory under cooled conditions $\left(4-8{ }^{\circ} \mathrm{C}\right)$ within of $24 \mathrm{~h}$ before processing. Samples for cultivation of anaerobes (dry wipes) were stored at $+4{ }^{\circ} \mathrm{C}$ for maximum of one week, whereas the samples for molecular analyses were immediately frozen $\left(-20^{\circ} \mathrm{C}\right)$ until processing.

\section{Bioburden assays}

Bioburden assays were performed as described in ECSSQ-ST-70-55C [13]. In brief, collected samples were extracted in sterile water (swabs) and PBS buffer incl. Tween $80(0.02 \%, w / v)$ (wipes) by a combination of vortexing and sonication. The remaining liquid was heatshocked at $80{ }^{\circ} \mathrm{C}(15 \mathrm{~min})$, afterwards plated on $\mathrm{R} 2 \mathrm{~A}$, and incubated for $72 \mathrm{~h}$ at $32{ }^{\circ} \mathrm{C}$. Appearing colonies were counted after 24, 48 and $72 \mathrm{~h}$.

During each sampling event, 26 wipe samples (D1D26) from cleanrooms and change room were taken (see Additional file 2: Table S2 and Fig. 1). Five field controls, and five laboratory controls were processed in parallel (Additional file 2: Table S2). Additionally, during the second, third and fourth sampling, spacecraft hardware was sampled with swabs and wipes, as given in full detail in Additional file 2: Table S2.

\section{Air samples}

During each sampling campaign, at least 10 air samples were taken, and at least two field controls, as well as laboratory controls were processed in parallel. Gelatine filters retrieved from the Sartorius AirPortMD8 device filter cassette were removed and immediately placed on an R2A agar plate. The colonies were counted after $72 \mathrm{~h}$ incubation at $32{ }^{\circ} \mathrm{C}$. Details on sampling locations are given in Additional file 3: Table S3.

\section{Biodiversity assays ("alternative assays")}

Wipe samples were extracted in PBS buffer incl. Tween $80(0.02 \%, w / v ; \mathrm{PBST})$ by a combination of vortexing and sonication [13]. The solution was, in $20 \mathrm{ml}$ aliquots, concentrated via filtration onto $0.45 \mu \mathrm{m}$ filters (Millipore, S-Pak-Filter, $47 \mathrm{~mm}$, sterile and made of hydrophilic polyvinylidene fluoride (PVDF)) under aseptic conditions. Each cultivation assay was performed in duplicates by placing the filters on (i) RAVAN agar incl. $50 \mu \mathrm{g} / \mathrm{ml}$ nystatin for oligotrophs [27] (modified: 1:100 diluted, final concentration of $50 \mathrm{mg} / \mathrm{l}$ sodium pyruvate instead of $20 \mathrm{mg} / \mathrm{l}$ pyruvic acid), (ii) $\mathrm{R} 2 \mathrm{~A} \mathrm{pH} 9$ for alkaliphiles (R2A, BD Difco; $\mathrm{pH}$ was adjusted with sterile $\mathrm{Na}$ sesquicarbonate), (iii) PDA for fungi (potato dextrose agar, BD) and (iv) R2A for vegetative mesophiles (R2A, BD Difco), respectively. Anaerobes were cultivated on TSA (trypticase soy agar) medium, but processed under strictly anoxic conditions, as described earlier [25]. Incubations were performed at $32{ }^{\circ} \mathrm{C} \pm 1{ }^{\circ} \mathrm{C}$ for up to 2 weeks (oligotrophs). The colonies were counted and reported at time points 24,48 and $72 \mathrm{~h}$ (vegetatives and fungi), 1, $3,5,7$ and 14 days (oligotrophs), and 1, 3, and 7 days (anaerobes). Details on the sampling locations etc. are given in Additional file 4: Table S4.

\section{Taxonomic analyses of the isolates by MALDI-TOF}

Selected isolates obtained from four sampling campaigns were processed at the premises of DSMZ (German Collection of Microorganisms and Cell Cultures, Braunschweig, Germany) and analysed by MALDI-TOF mass spectrometry. MALDI-TOF analysis was used for grouping the strains into various clusters and to identify the isolates to species level. Colony morphology of all strains was checked from subcultures grown on agar plates. Routine media used for subculturing included R2A and TSA. Cell morphology was examined from liquid cultures by using phase-contrast microscopy (Zeiss Axioscope A.1, $\times 100$ Plan-Neofluar oil-immersion objective, Ph3; Zeiss Axiocam MRc, and Software Axiovision). Agar slides were coated with a layer of highly purified agar $(2 \%)$ and $20 \mu \mathrm{l}$ of the freshly grown liquid culture was dropped onto the agar layer and spread by a cover slip. Aliquots from pure cultures were stored in Microbank tubes (Prolab Diagnostics) for short term storage. Pure cultures were characterised by MALDI-TOF, and when needed identified by $16 \mathrm{~S}$ rRNA gene sequencing or automated ribotyping in addition.

MALDI-TOF sample preparation followed protocol 3 as described [28]. After ethanol-formic acid extraction, $1 \mu \mathrm{l}$ of supernatant was transferred onto a target plate and allowed to dry in air at normal room temperature. Subsequently the sample was overlaid with $1 \mu$ l of the matrix solution and air-dried again. MALDI-TOF mass spectrometry was conducted using a Microflex L20 mass spectrometer (Bruker Daltonics) equipped with a N2 laser. Spectra were collected as a sum of 500 shots across as spot. A mass range of 2000-20,000 m/z was used for analysis. Spectra obtained for all isolates were analysed, and compared with reference spectra from the database for identification using the BioTyper software (Bruker Daltonics). Riboprinting was applied using the automated Riboprinter microbial characterisation system (Dupont, Qualicon). Sample preparation and analysis were performed according to the manufacturer's instructions and EcoRI restriction enzyme was used to generate the DNA fragments.

Maintenance of cleanroom isolates for long term storage Cryopreservation in glass capillaries was used for longterm storage of chosen cleanroom isolates, which were previously selected from the MALDI-TOF groups. Freshly grown cells were harvested by centrifugation and resuspended in a small aliquot of the sterile medium 
(1 ml) containing a cryoprotectant. The capillaries were filled by using a micropipetting aid and subsequently flame sealed on both ends. Glycerol or dimethyl sulfoxide (DMSO) may be used as cryoprotectant. The final concentration of glycerol is usually $10-15 \%(v / v)$. DMSO is used at a final concentration of $5 \%(\mathrm{v} / \mathrm{v})$. DSMO may be either filter sterilised or autoclaved under a nitrogen atmosphere at $115{ }^{\circ} \mathrm{C}$ for $15 \mathrm{~min}$. A detailed protocol of this technique is publicly accessible at www.cabri.org. Prepared glass capillaries were placed in a storage container and laid in the gas phase of the liquid nitrogen tank first. Once frozen, the storage container was placed on its selected position in the tank.

\section{Molecular assays}

During each sampling, 10 wipe samples for molecular analyses were taken. A list of these samples is given in Additional file 5: Table S5, and sampling locations are indicated in Fig. 1.

Due to the low biomass, three molecular samples taken from each cleanroom during each sampling campaign were pooled, as were the extraction blanks. Samples from change room and field blank were processed individually. The wipes were transferred into DNA-free bottles (baked at $250{ }^{\circ} \mathrm{C}$ for $24 \mathrm{~h}$ ) filled with of PCR grade water. The bottles were sonicated for $120 \mathrm{~s} \pm 5 \mathrm{~s}$ with a maximal power of $240 \mathrm{~W}$ and a frequency of $40 \mathrm{kHz}$, and vortexed at maximum speed for $1 \mathrm{~min}$. The biomass-containing water suspension was concentrated to 200-500 $\mu \mathrm{l}$ using UV sterilised Amicon filters (Amicon Ultra $15 \mathrm{ml}, 50 \mathrm{~K}$, Merck Millipore). DNA extraction was performed using a modified XS-buffer method [24] with a bead beating step to disrupt thick-walled microorganisms such as bacterial spores, using beating tubes included in the MO BIO Power Biofilm ${ }^{\mathrm{TM}}$ DNA Isolation Kit (Carlsbad, CA, USA).

For molecular cloning, near full length $16 \mathrm{~S}$ rRNA gene was amplified using the TaKaRa Ex Taq polymerase (Clontech, Japan) with the primer 9bF [29] and 1406uR [30] under the following PCR conditions: initial denaturation at $95^{\circ} \mathrm{C}$ for $2 \mathrm{~min}$, followed by 30 cycles of denaturation $96{ }^{\circ} \mathrm{C} 30 \mathrm{~s}$, annealing $60{ }^{\circ} \mathrm{C} 30 \mathrm{~s}$, extension $72{ }^{\circ} \mathrm{C}$ $60 \mathrm{~s}$, and a final extension at $72{ }^{\circ} \mathrm{C} 10 \mathrm{~min}$. PCR products were visualised on a $1.5 \%$ agarose gel. $1 \mu \mathrm{l}$ of each PCR product was cloned in StrataClone SoloPack competent cells (Agilent Technologies, StrataClone PCR Cloning Kit) according to manufacturer's instructions. Depending on the concentration of the PCR product, 24-96 clones of each sample were sent to Macrogen in Amsterdam for unidirectional sequencing (primer 1406uR; [30]). In addition, the community composition and diversity of Bacteria and Archaea in ExoMars cleanrooms was studied using next generation sequencing (NGS; Illumina MiSeq). For this approach, variable region V4 of $16 \mathrm{~S}$ rRNA gene was amplified with "universal" PCR primers 515F (5'GTGCCAGCMGCCGCGGTAA-3') and 806R (5'GGACTACHVGGGTWTCTAAT-3') [31] using TaKaRa Ex Taq polymerase (Clontech, Japan). Cycling conditions consisted of an initial denaturation at $94{ }^{\circ} \mathrm{C}$ for $3 \mathrm{~min}$, followed by 35 cycles of denaturation $94{ }^{\circ} \mathrm{C} 45 \mathrm{~s}$, annealing $50{ }^{\circ} \mathrm{C} 60 \mathrm{~s}$, extension $72{ }^{\circ} \mathrm{C} 90 \mathrm{~s}$, and a final extension at $72{ }^{\circ} \mathrm{C} 10 \mathrm{~min}$. The produced fragments were sequenced at ZMF Core Facility Molecular Biology in Graz, Austria, using the available Illumina MiSeq platform.

\section{Sequence data analysis of cloning and next generation sequencing}

To analyse the microbial community composition and taxonomic diversity obtained raw reads were processed using mothur version 1.36.1 [32] following the Standard Operation Procedure (SOP): For MiSeq data, the paired end reads were joined together, and the produced sequences were quality checked (minimum length 200, maximum length 300, maximum number of homopolymers 8) and aligned against mothur formatted SILVA 123 database [33]. Then, the good quality sequences were pre-clustered and chimeric sequences were removed. The number of sequences per sample before and after quality control was following: September 13 change room: before qc 61,610/after qc 40,831, September 13 cleanroom 4a: 47,961/30141, September 13 cleanroom 02: 49,332/30093, December 14 change room: 47,036/ 30127, December 14 cleanroom 4a: 55,643/38875, December 14 cleanroom 02: 60,188/38046, February 15 change room: 50,252/33269, February 15 cleanroom 4a: $76,842 / 39656$. Taxonomic assignment was performed by querying the sequence reads against a trainset14_032015 reference database, and the sequences were clustered into OTUs (threshold 0.03 dissimilarity) using average neighbour algorithm. A biom table was constructed for downstream analyses, and OTUs represented by 5 or less sequences were removed. The Sanger data was processed similarly, except for merging the raw reads and quality check in the beginning, as the raw data was received in fasta format. These data processing steps were performed in Galaxy, which is an open source webbased platform for data processing and analysis [34]. This platform was made available by the Center for Medical Research (ZMF), Medical University of Graz. To further analyse these datasets, to calculate alpha and beta diversities, differences in community composition, and visualise the results, we applied Calypso (Version 5.8 ), an online platform for mining, visualising and comparing multiple microbial community composition data (cgenome.net/calypso). Total-sum normalisation was applied for $16 \mathrm{~S}$ rRNA gene data. For network and functional analyses we only used the MiSeq data. To create the networks, OTUs were clustered and weights were 
calculated using a stochastic spring-embedded algorithm. The resulting edge and node tables were visualised using Cytoscape 2.8.3 [35]. Here, the OTUs were coloured by their sample origin and their relative abundance was correlated with the node size. To analyse the predicted functions of studied microbial communities, the sequence data was processed with Qiime [36] openreference OTU picking pipeline with GreenGenes taxonomy (13_8 database [37]), and a PICRUSt (version 1.0.0.) analysis was performed using the default settings [38]. Text formatted biom tables of MiSeq and Sanger sequence data are given in the Additional file 6: Table S6 and Additional file 7: Table S7. Details about data analysis pipelines are given in Additional file 8. Sequence data were submitted to the European Nucleotide Archive (ENA) with the study accession number PRJEB15908.

\section{Quantitative real-time PCR}

Ribosomal RNA gene copy numbers were quantified with quantitative real-time PCR (qPCR) in triplicates using the RotorGene 6000 Real-Time PCR system (Corbett Life Science, Concorde, NSW, Australia). The qPCR reactions were carried out in volume of $10 \mu \mathrm{l}: 5 \mu \mathrm{l} 1 \mathrm{x}$ SYBR Green Taq Premix (Quantitect SYBR Green PCR kit, Qiagen, Hilden, Germany), $1 \mu \mathrm{l}$ of each primer $(3 \mu \mathrm{M}), 2 \mu \mathrm{l}$ water (Merck Millipore), and $1 \mu \mathrm{l}$ of extracted DNA template. To quantify Bacteria we applied Bacteria specific primers 338bF and 517uR [30]. Purified standard of Desulfovibrio desulfuricans and negative controls (no template) were included in all qPCR runs. The quantification of standard was performed with the Qubit Quantitation Platform 2.0 (High Sensitivity Kit, Invitrogen, Carlsbad, CA, USA). Cycling conditions for qPCR consisted of an initial denaturation at $95{ }^{\circ} \mathrm{C}$ for $15 \mathrm{~min}$ and a cycling protocol as follows: denaturation at $94{ }^{\circ} \mathrm{C}$ for $15 \mathrm{~s}$, annealing at $60{ }^{\circ} \mathrm{C}$ for $30 \mathrm{~s}$ and elongation at $72{ }^{\circ} \mathrm{C}$ for $30 \mathrm{~s}$. Melting curve was generated at $72-95{ }^{\circ} \mathrm{C}$. Copies detected in qPCR negative controls were subtracted from sample values.
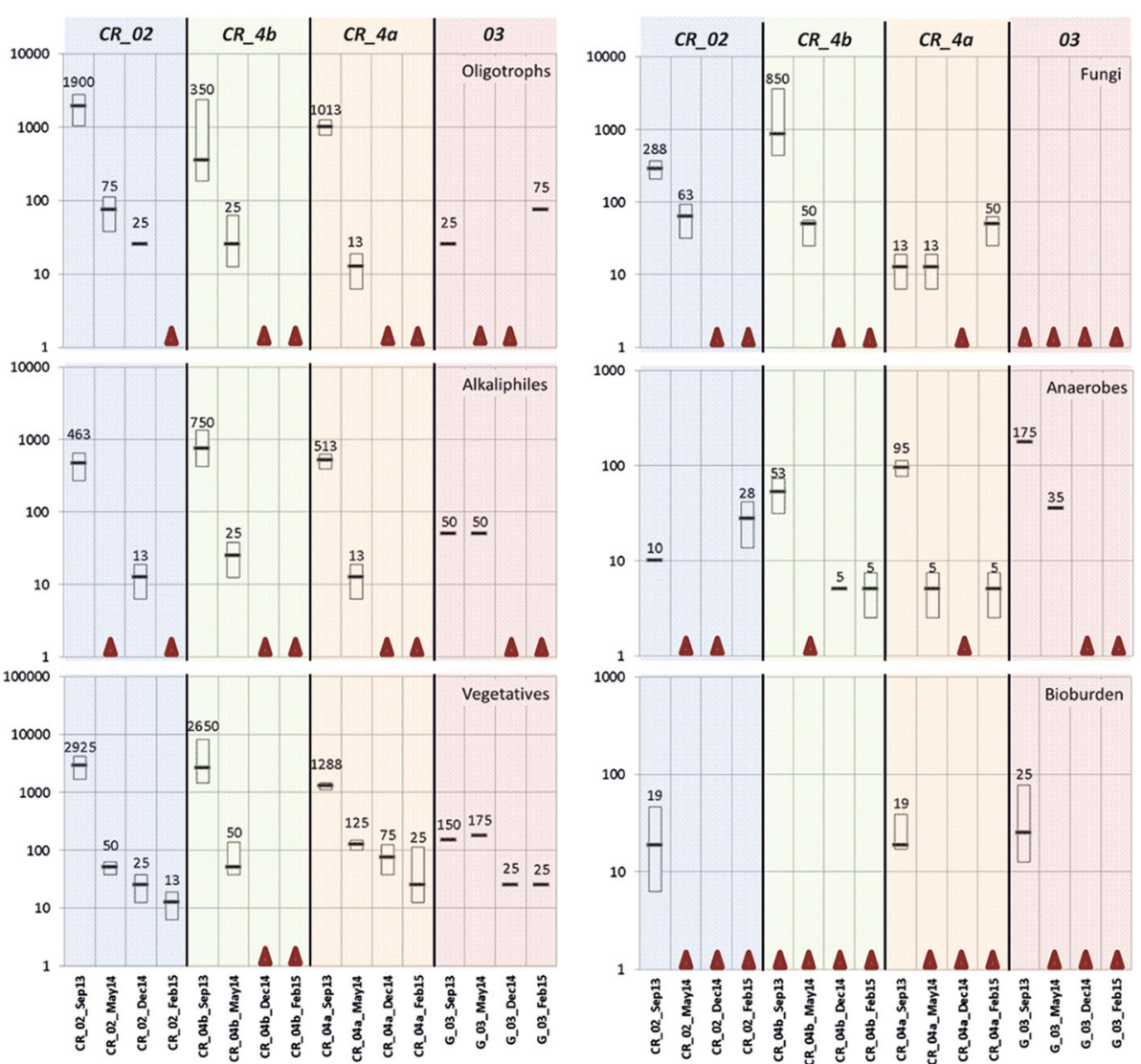

Fig. 2 Retrieved colony forming units from alternative assays (oligotrophs, alkaliphiles, vegetatives, fungi and anaerobes) and bioburden measurements, grouped by sample origin. Horizontal lines and given numbers reflect the median of all samples taken at this certain timepoint in the respective cleanroom (source: Additional file 2: Table S2 and Additional file 4: Table S4). Boxes represent the first and third quartile. Red triangles refer to a median of 0 . Colony forming units (CFUs) are given in a logarithmic scale ( $Y$-axis). Colours indicate the different sampling campaigns, sampling locations are indicated ( $X$-axis) 


\section{Results}

Bioburden assays reflect the consequent reduction of microbial contamination throughout the sampling period, reaching the detection limit at the last sampling

The bioburden assays aimed at the detection of heatshock resistant microorganisms that are considered to represent the most harmful microbial contamination source for planetary protection issues. In detail, the median of colony forming units (CFUs) in cleanrooms $02,4 a, 4 b$ and change room 03 , was zero throughout the time the spacecraft hardware was in the facility (Fig. 2). No colonies were retrieved from samples taken during the February 2015 (last) sampling and thus our methods reached the detection limit. Before AIT activities started, the median of the bioburden was higher in cleanrooms 02 and $4 \mathrm{a}$, as well as in the garment room $03(19,19$, and 25 CFUs per $\mathrm{m}^{2}$, respectively) (Fig. 2, Additional file 2: Table S2). The strict cleaning and management regime of the cleanroom complex was found to result in a substantial reduction of the bioburden. Swab and wipe samples taken from spacecraft hardware revealed zero CFUs for the last sampling and extremely low counts for the other two samplings before (Additional file 2: Table S2). Only a few microorganisms were retrieved from heatshocked samples taken from spacecraft hardware.

\section{Overall cultivable diversity decreased during AIT activities} The biodiversity assays aimed at the cultivation of a broader diversity of microorganisms from the cleanroom facility, including oligotrophs, alkaliphiles, vegetatives, fungi and anaerobes [18, 23, 25, 39].

The highest median CFU counts were observed for vegetative microorganisms, which remained detectable also during the last sampling event (Additional file 4: Table S4, Fig. 2). However, the microbial contamination load was substantially reduced compared to the first sampling, when spacecraft hardware had not entered the cleanrooms yet. Notably, no CFUs were observed on PDA agar ('Fungi') within the change room (room 03), throughout the sampling period, although bacteria were found to be present therein. Overall, the OTUs retrieved from alternative assays (as well from air samples, Additional file 3: Table S3), revealed a decreased amount of cultivable microorganisms in the course of AIT activities, confirming the increased microbial cleanliness observed by bioburden measurements.

\section{MALDI-TOF mass spectrometry allowed the reliable identification of microbial isolates}

A total of 113 isolates originally obtained during the sampling period were analysed by MALDI-TOF mass spectrometry. In case that identification of a cleanroom isolate failed using MALDI-TOF, characterisation was completed by $16 \mathrm{~S}$ rRNA gene sequencing or automated ribotyping. A summary of all isolates data is shown in Additional file 9: Table S8, and a dendrogram of identified isolates is given in Additional file 10: Figure S1. Selected isolates were assigned a DSM number and are now available through the ESA catalogue at DSMZ (https:/www.dsmz.de/research/microorganisms/projects/ european-space-agency-microbial-strain-collection.html).

Arthrobacter, Bacillus, Micrococcus and Staphylococcus were the most abundant isolates obtained and processed (Additional file 9: Table S8). Whereas Bacillus and Staphylococcus species were obtained from a number of surfaces and under different enrichment conditions, Arthrobacter was detected during the first sampling only, and solely on cleanroom surfaces. Within the bioburden studies (see Additional file 11: Table S9.) endospore forming bacteria Bacillus mycoides, B. subtilis and B. megaterium where the only retrieved contaminants from the cleanroom areas during all sampling campaigns, and only few microorganisms were retrieved from heat-shocked samples taken from spacecraft hardware, namely Staphylococcus pasteuri, S. warneri, S. sp., Rothia amarae, B. subtilis and B. mycoides (Additional file 11: Table S9). The Staphylococcus species are clearly associated with the human body as its source. B. mycoides and B. subtilis were the only spore-forming microorganisms retrieved from spacecraft hardware. Interestingly, isolates enriched in September 2013 and December 2014 were found to be related to the Bacillus subtilis group, but strains obtained in February or May prevalently to B. mycoides, B. pumilus, or B. simplex (Additional file 11: Table S9). Although the $B$. mycoides strains were isolated from different locations, the riboprint pattern obtained for seven of the isolates could be assigned to the same ribogroup, indicating that one strain might be spread out within cleanroom $4 \mathrm{~b}$ (Additional file 12: Figure S2).

The highest diversity in general was detectable within the samplings obtained in September 2013 (19 genera), followed by samples received in May 2014 (8 genera). Sixteen bacterial genera were cultivated on R2A agar from sampling campaign in September 2013 (Agrococcus, Arthrobacter, Bacillus, Brevibacterium, Brevundimonas, Cryptococcus (yeast), Hymenobacter, Kocuria, Massilia, Methylobacterium, Paenibacillus, Paracoccus, Pedobacter, Rathayibacter, Rhodosporidium, Staphylococcus), whereas only 6 of these, namely Bacillus, Kocuria, Methylobacterium, Paenibacillus, Paracoccus and Staphylococcus species were observed during AIT activities. Isolates related to Arthrobacter and Hymenobacter could only be detected in samples taken in September 2013, and Methylobacterium and Kocuria occurred in May 2014. Micrococcus luteus and Paracoccus yeei were predominant in February 2015. Staphylococci were detectable in all samplings, but mostly in September 2013, May and December 2014. 12 isolates were identified as Staphylococcus hominis and three 
isolates as $S$. warneri. Only a few isolates could been assigned to the species $S$. aureus, S. epidermidis, $S$. saprophyticus, S. lugdunensis or S. simulans.

Micrococcus was obtained during three of four sampling events, and was mainly detected on cleanroom surfaces. Both species, Arthrobacter and Micrococcus, preferred specific enrichment media, namely oligotrophic and alkaliphilic conditions. Those microbes, as many others, where not detected when a heatshock was applied to the sample (bioburden assays).

Fungal genera, namely Trichoderma, Gibellulopsis, Alternaria and Lecythophora, were only found in cleanroom 02 in September 2013, whereas different yeast species (see Additional file 11: Table S9) were found in cleanrooms 02 and 04b. A broad microbial diversity was retrieved from RAVAN agar, targeting microorganisms that can grow under extreme nutrient constraints, including Kocuria, Micrococcus, Microbacterium, Arthrobacter, Rathayibacter, Curtobacterium (all Actinobacteria), Bacillus (Firmicutes), Paracoccus, Methylobacterium, Roseomonas, Sphingomonas (all $\alpha$-Proteobacteria), Hymenobacter (Bacteroidetes) and Pigmentiphaga ( $\beta$-Proteobacteria). Strictly and facultatively anaerobic microorganisms were enriched on TSA plates that were incubated under anoxic conditions. In particular, Staphylococcus, Bacillus, and Dermabacter species were enriched by this method, representing facultatively anaerobic bacteria. During the last, cleanest sampling, only Bacillus, Micrococcus, Paracoccus (three strains) and Staphylococcus were grown.

\section{Results from quantitative PCR indicate the presence of} DNA signatures from dead cells in the cleanroom areas Although bioburden and alternative measurements revealed a subsequent reduction of the microbial contamination, in particular during AIT activities, the results from quantitative PCR were less informative. The lowest contamination with $16 \mathrm{~S}$ rRNA gene copies was found in cleanroom 4b throughout the analysed time frame (range from below the detection limit (BDL) to 23.204 copies $/ \mathrm{m}^{2}$ ). However, the clear trend of decreasing microbial load could neither be followed in the cleanrooms (range from BDL to 49.477 copies $/ \mathrm{m}^{2}$ ), nor in the change room (range from BDL to 99.690 copies $/ \mathrm{m}^{2}$ ) (Additional file 13: Figure S3).

\section{Sanger sequencing results display a strong impact of human associated bacteria on cleanroom microbiota}

We also studied the cleanroom microbial communities using Sanger sequencing, following cloning of the $16 \mathrm{~S}$ rRNA gene pool. In total, we identified 70 different bacterial taxa. Several human skin associated bacteria were abundant in the dataset: Propionibacterium was the most abundant genus, and always present in change rooms, as well as in cleanrooms in September 2013, before the AIT activities started. Propionibacterium was also the only genus that was statistically significantly increased in change room compared to cleanrooms (analysis of variance, $p \leq 0.05$, rank test, $p=0.027$ ). Additionally, other human and human skin associated bacteria, such as Streptococcus, Staphylococcus and Corynebacterium tended to be more abundant or solely present in the change room. Additionally, Streptococcus and Sphingomonas were more abundant before the AIT activities started (Bayesian analysis of variance, $p \leq 0.05$ ). At all sampling campaigns, cleanrooms carried more diverse bacterial communities compared to change room (Fisher's alpha index, $p=0.01$ ), and the cleanrooms were most rich in microbial diversity before the AIT activities started, further strengthening the observation of decreasing contamination towards the end of project. The 20 most abundant bacterial genera in Turin cleanrooms based on cloning and Sanger sequencing are presented in Fig. 3.

\section{Next generation sequencing uncovers the diversity and divergence of cleanroom microbial communities}

We further characterised the microbial communities in ExoMars cleanrooms using Illumina MiSeq next generation sequencing. In the whole dataset, we identified 15 microbial phyla, of which 12 were affiliated to Bacteria and 3 to Archaea (Thaumarchaeota/Nitrososphaera, Euryarchaeota/Pyrococcus and unclassified Archaea). The most abundant bacterial phyla were Proteobacteria (76\% of all sequence reads), Firmicutes (11\%), Actinobacteria (10\%), and Bacteroidetes (2\%). The most abundant identified genera were Cupriavidus (41\% of all sequence reads), Pseudomonas (10\%) Staphylococcus (7\%), Corynebacterium (6\%), and Delftia (3\%). Sphingomonas and Acinetobacter were detected at $1 \%$ relative abundance. All other taxa were represented by less than $1 \%$ of the total sequence reads.

The diversity of cleanroom microbial communities changed in the course of the project. On the first sampling campaign, before the start of AIT activities, microbial $\alpha$-diversity in cleanrooms was relatively high (inverse simpson index 24-26). The change room carried considerably less diversity, and after changing the cleaning solutions before the next sampling campaigns, the diversity also decreased in cleanrooms and stayed below the approved limit throughout the project.

We also compared the community structures of cleanrooms and change room at different sampling time points ( $\beta$-diversity). Cleanrooms and change room carried distinct microbial communities: principal component analysis (Fig. 4) demonstrates that especially in the beginning of the study in September 2013, the change room groups far from other sampling time points and locations, indicating a distinct community structure. The 


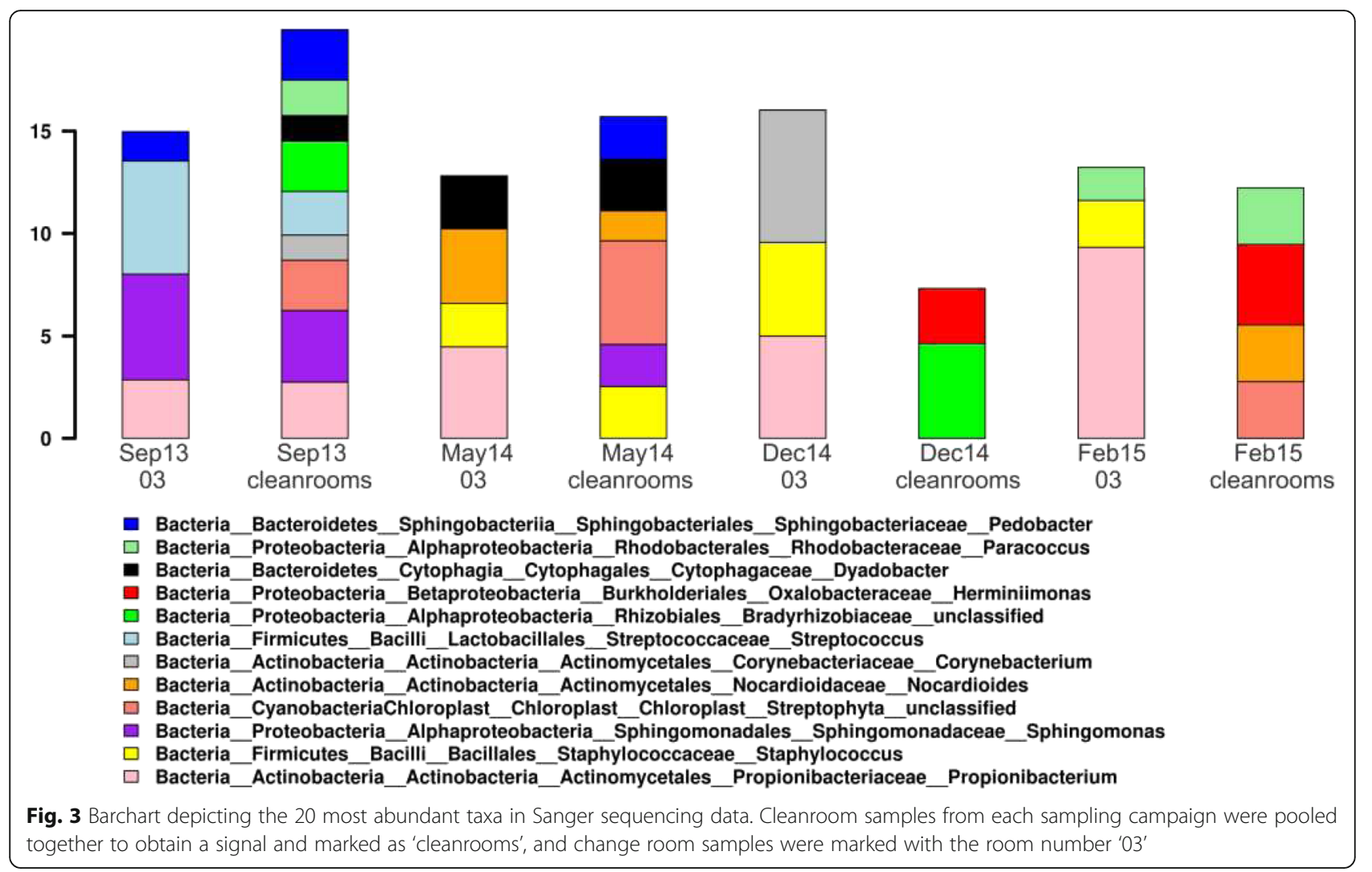

cleanroom samples collected on the same day were always highly similar and grouped together, and the samples gathered in separate time points were always different to certain extent. Furthermore, the principal component analysis comparing the microbial communities before and after the AIT activities started shows that there is a shift in the community structure after the hardware were brought in the cleanroom and the cleaning regime changed, suggesting that the microbial community is determined by external factors, such as the work conducted in cleanroom, personnel, and cleaning regime.

The identified taxa were also compared with the linear discriminant analysis (LDA) effect size method (LEfSe) [40] to find those taxa that are significantly different between the compared sample groups. Human skin
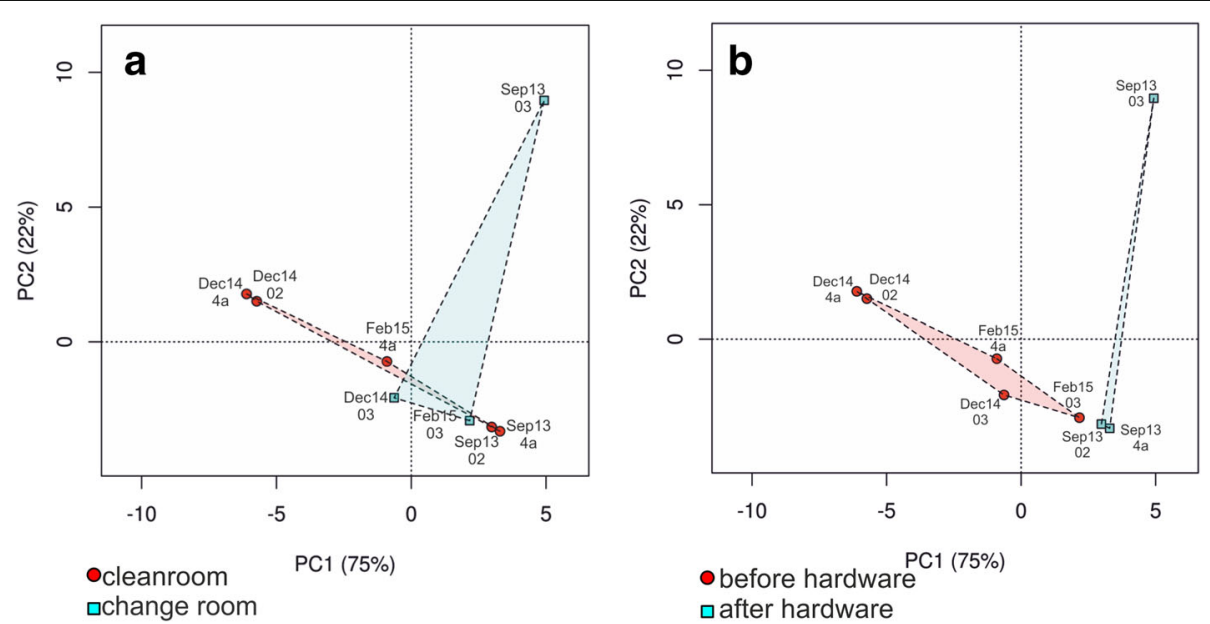

Fig. 4 PCA plot depicting the relationships of cleanroom and change room microbial communities (a) and communities before and after the spacecraft hardware was brought in (b) based on taxonomy information 
associated Staphylococcus and a soil bacterium Mucilaginibacter were more abundant before the AIT activities started, and Cupriavidus, the highly resistant bacterium, was more abundant after the hardware were brought in and the cleaning regime changed to more efficient (LEfSe, $p \leq 0.05$ ).

\section{Predicted functions indicate an elevated stress level for microbial inhabitants during AIT activities}

We applied PICRUSt [38] to predict the microbial genes present in the ExoMars cleanroom based on MiSeq amplicon data. In general, the most abundant identified functions were involved in membrane functions, such as transport systems and receptor proteins, quorum sensing and environmental information processing.

We compared the community functions between cleanrooms and change room, and before and after the start of AIT activities in order to assess if the level of confinement and mode of use affected the predicted community function. Principal components analysis plots visualise the distinct communities between cleanrooms and change room throughout the the ExoMars assembly, and between the first sampling and after the hardware were in the cleanrooms (Additional file 14: Figure S4).

The identified functions were also compared with the linear discriminant analysis (LDA) effect size method (LEfSe) [40] to find the most differentially abundant functions between cleanrooms and change room and before and after AIT activities were initiated. Several predicted genes were more abundant in change room, including genes coding for base excision repair protein exodeoxyribonuclease, and several genes involved in membrane functions. In cleanrooms, motility associated genes, such as pilus assembly protein and chemosensory two-component regulatory system were more abundant.

We also studied how the initiation of AIT activities and change in cleaning regime affected the microbial communities at functional level. The results show that functions related to nucleotide excision repair, $A B C$ transporters, quorum sensing, and metabolism of terpenoids and polyketides, as well as biodegradation and metabolism xenobiotics increased in relative abundance after the activities started and the cleanroom was cleaned with more effective cleaning products.

\section{AIT activities have tremendous impact on the microbial community composition and the microbial transfer from one room to another}

The two representative network analyses (before and during the cleanroom complex harboured spacecraft hardware) show a different picture of the microbial community in the single rooms and the overlap of the found taxonomies. Notably, the change room harbours a less unique microbial community during the first sampling in
September 2013, whereas the cleanroom areas 02 and 4a reveal quite a number of microbial signatures unique to their location. The picture is drastically different in December 2014, where the change room harbours the largest number of unique taxons (Fig. 5; Additional file 15: Figure S5 and Additional file 16: Figure S6). The core microbiota of all three areas was composed of signatures from, e.g. Pelomonas, Sphingomonas, Methylobacterium, Pseudomonas, Cupriavidus, Escherichia/Shigella, Micrococcus, Brevundimonas, Corynebacterium, Delftia, Acinetobacter, Staphylococcus, Enhydrobacter (September 2013), and Delftia, Micrococcus, Staphylococcus, Rhizobium, Cupriavidus, Escherichia/Shigella, Enhydrobacter, and Sphingomonas (December 2014), with those printed in bold letters appearing in both core microbiotas (Additional file 15: Figure S5 and Additional file 16: Figure S6). Cupriavidus was the most prominent taxon found in the core microbiota of the December 2014 sampling. During December 2014 , only four microbial taxa were found to be distributed from cleanroom 02 and $4 \mathrm{a}$ (besides the core taxa) and vice versa, namely unclassified Sphingomonades, Massilia, Pseudomonas and unclassified Betaproteobacteria. All in all, the network analyses confirmed the extraordinary efficiency of the cleanliness regimes during the AIT activities and the presence of the spacecraft hardware in the rooms.

\section{Variety of methods generates unique information on the cleanroom microbial communities}

We analysed the microbial community structure in ExoMars cleanrooms using three methods: MiSeq amplicon sequencing, cloning and Sanger sequencing, and cultivation. We expected merely partially overlapping results as the applied methods differ considerably. Overall, 179 different bacterial genera were detected during the entire study. With high throughput MiSeq amplicon sequencing we detected majority of these taxa (88\%), but both Sanger sequencing and cultivation produced unique information on the cleanroom microbial communities. Pigmentiphaga, Dermabacter, Luteimonas, and Agrococcus were detected only via cultivation, and 16 different taxa via Sanger sequencing, inluding Brochothrix, Oligotropha, Gemmatimonas, Dehalobacter, Pelomonas, Pseudoclavibacter, Variovorax, Parabacteroides, and Lactococcus. Only 12 taxa (7\%) were identified with all three methods: Brevundimonas, Micrococcus, Staphylococcus, Pedobacter, Microbacterium, Massilia, Bacillus, Sphingomonas, Paenibacillus, Methylobacterium, Hymenobacter, and Paracoccus. Venn diagram (Fig. 6) depicts the overlap of identified bacterial genera with used analysis methods. List of all detected taxa and analysis methods can be found in Additional file 2: Table S2, Additional file 3: Table S3, Additional file 4: Table S4, Additional file 5: Table S5, Additional file 6: Table S6, Additional file 7: Table S7, Additional file 9: Table S8 and Additional file 11: Table S9. 


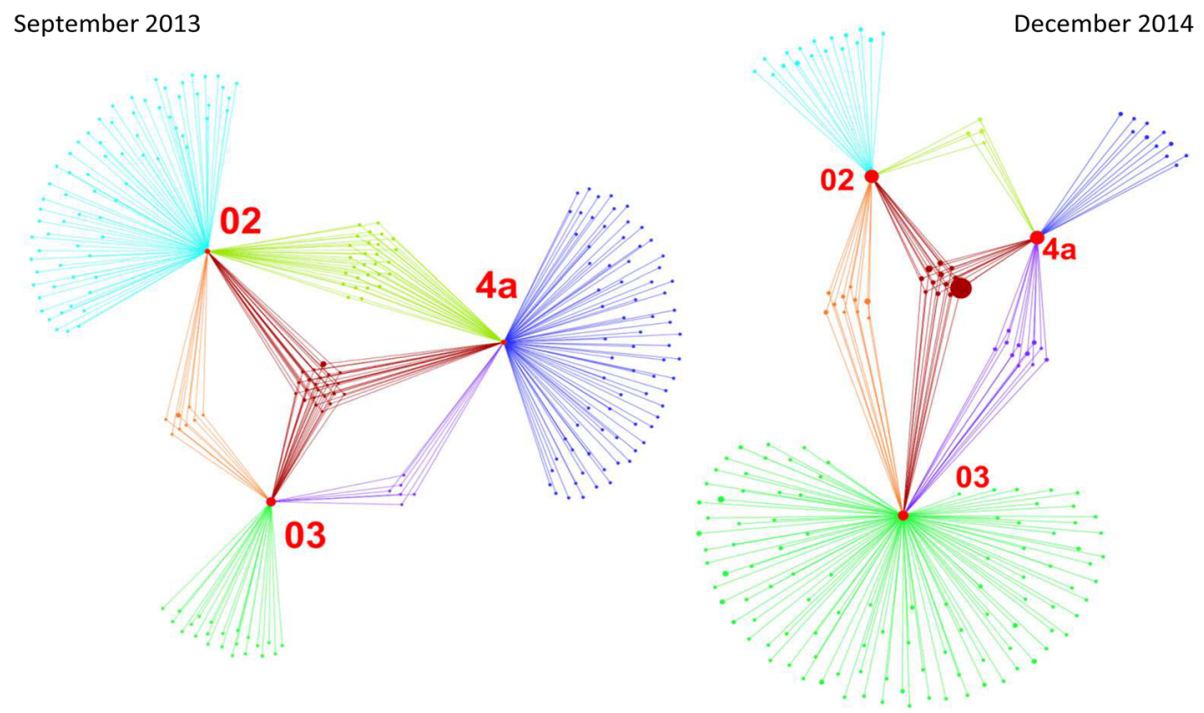

Fig. 5 Cytoscape network for two representative sampling events: September 2013 (before AIT) and December 2014 (cleanroom harboured spacecraft hardware). 02 and $4 a$ refer to cleanroom areas, whereas 03 is the changing room. A more detailed picture of the two networks, including taxonomies, is given in Additional file 15: Figure S5 and Additional file 16: Figure S6

\section{Discussion}

ESA's ExoMars 2016 mission reached Mars in the middle of October 2016. The Schiaparelli module touched down on October 192016 on Meridiani Planum, a flat, even region on Mars. Due to planetary protection constraints, the spacecraft hardware was assembled under biological contamination control in specifically designed and built cleanrooms. The results confirm that the bioburden, detected microbial contamination, and microbial

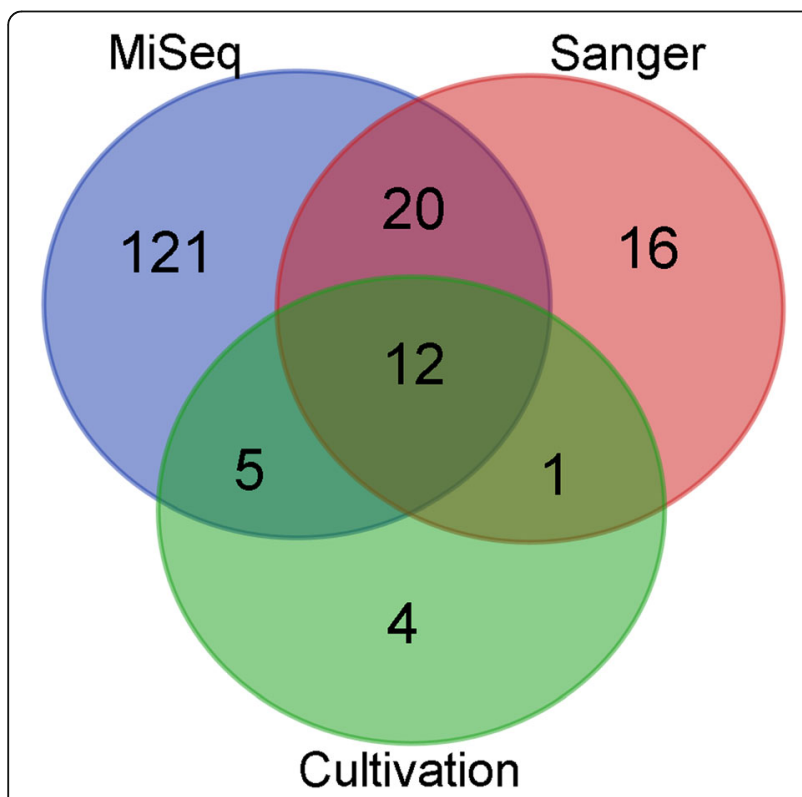

Fig. 6 Venn diagram visualising the overlap between identified microbial genera detected in this study by cultivation, MiSeq and Sanger sequencing diversity in cleanrooms decreased during the assembly, integration and testing period after the cleanroom was decontaminated with highly effective cleaning agents and alternating $\mathrm{pH}$. At no point, the spacecraft hardware showed microbial contamination levels above the expected and acceptable limit.

For the first time, the isolated microorganisms from cleanrooms and spacecraft hardware were identified using MALDI-TOF, which was found to be a very powerful tool for taxonomic determination of planetary protectionassociated microbial isolates. MALDI-TOF analysis of bacterial cells is well-recognised as a fast and reliable tool for rapid characterisation and identification of bacteria, because the spectra obtained from the vegetative bacterial cells are dominated by peaks of the ribosomal proteins. These proteins are conservative molecules and best suited for classification and identification of bacterial species. As shown by Schumann and Meyer [28] changes in the cell architecture of endospore-forming bacteria, caused by sporulation, may significantly alter the MALDI-TOF mass spectra by detection of spore proteins. As a consequence, the successful identification of Bacillus and related species by MALDI-TOF requires that the culture under question is analysed in the same physiological state for which the database entry was generated. Following this requirement, reliable protein based differentiation of Bacillus isolates by MALDI-TOF was exemplary shown for the Bacillus pumilus $[41,42]$. In our study, the identity of Bacillus isolates was additionally confirmed via riboprinting and $16 \mathrm{~S}$ rRNA gene sequencing. Our results from the bioburden assays performed for the ExoMars 2016 mission were in accordance with the results retrieved from 3000 independent 
samples, analysed by the microbiology laboratory run by Thales Alenia Space in Turin.

A number of representatives of microorganisms, that were isolated also during this study, including $B$. cereus [43], B. megaterium [44], B. mycoides [45], B. pumilus [46], B. subtilis [47-51], Micrococcus luteus [52] and Staphylococcus aureus [53] have been tested for the potential to survive space flight, or their capability to survive under simulated Mars conditions. In particular, $B$. subtilis (spores) has been found to be able to survive spaceflight, including impact, planetary ejection and atmospheric re-entry. B. mycoides is typically found in soil [54], but has been found to survive Martian simulations. Notable, B. mycoides isolates were retrieved several times during this study, and riboprinting indicated that the B. mycoides isolates (May 2014) belonged to the same ribogroup, supporting the assumption, that one strain was spread in the cleanroom $4 \mathrm{~b}$.

Bioburden assays, aiming to detect heat-shock surviving microorganisms did not only detect spore-forming microbes that are considered to represent the most harmful microbial contamination source for planetary protection issues, but also non-spore formers: we additionally retrieved, e.g. seven different Staphylococcus species from different areas. Staphylococcus is a typical human-associated bacterium, as were many other isolates identified in this study. Also a large proportion of bacterial signatures detected by molecular approaches were associated to the human body. We detected for example Propionibacterium, an anaerobic to microaerophilic chemo-organotrophic bacterium which lives in sweat glands, sebaceous glands, and other areas of the human skin, to be more abundant in the change room compared to cleanroom. Additionally, Streptococcus, Staphylococcus and Corynebacterium, belonging to the human microbiome, living in mouth, intestine, upper respiratory tract, and on skin, were abundant particularly in the change room which reflects the human influence in the garment area microbiota. Although the human influence, and thus the spreading of human-associated microorganisms is limited to a minimum, these microorganisms are the most abundant contaminants in cleanrooms, similarly as in other confined environments [9]. Additionally, we found environmental bacteria, including Cupriavidus, a chemoorganotrophic/chemolithotrophic, metal-resistant bacterium adapted to survive in harsh conditions, a strictly aerobic Delftia, which can cause human infections, as well as Acinetobacter, an environmental bacterium that can also reside on the human skin.

A number of microorganisms, including Microbacterium, Bacillus, Methylobacterium, Hymenobacter and Paracoccus were detected with all methods used in this study. Hymenobacter is often associated with cold environments [55, 56] and Microbacterium has been isolated from human clinical samples [57]. These bacteria were identified solely in first sampling. Soil and plant associated Methylobacterium [58] was identified in first and second sampling, and spore-forming Bacillus recurrently in all samplings. Additionally, Paracoccus yeei was found frequently in two sampling events, and in particular during the last sampling. P. yeei is known as a typical environmental microorganism, thriving in soil and brines, but has also been associated with unusual (skin) and eye infections in the human body $[49,50]$. Interestingly, representatives of all these genera have recently been identified as highly resistant against UV-C radiation and desiccation [59] which could explain their success also in the cleanroom. Successful isolation of all these bacteria suggests that they are able to endure or even thrive in the harsh cleanroom conditions, which are known to pose extreme stress and selection pressure on the microbial inhabitants, not only due to strict cleaning and decontamination procedures, but also the lack of water, nutrients and cofactors, as has been indicated in previous studies of cleanroom associated microbial communities $[10,22,60]$. As a consequence, although the number of microorganisms is tremendously reduced by frequent cleaning procedures and decontamination, the remaining bacteria are typically survival specialists with various resistances against one or several stressors, including antibiotics, metals, radiation, desiccation, or starvation. Interestingly, most of our cultivated bacteria, namely genera Arthrobacter, Bacillus, Brevibacterium, Brevundimonas, Curtobacterium, Kocuria, Massilia, Methylobacterium, Micrococcus, Microbacterium, Paenibacillus, Paracoccus, Pedobacter, Roseomonas and Sphingomonas have previously been identified as contaminants in sequence-based microbiota analyses [61]. This finding indicates that the DNA contamination of kits or laboratory reagents may be caused by resistant bacteria that can survive in extremely severe environments.

Overall, all cultivation methods applied in this study indicated a very clear trend during the AIT activities towards a reduction of the microbial contamination under detection limit (bioburden assays) or a very low level (biodiversity assays). These findings were confirmed by molecular analyses, including cloning/Sanger sequencing, and next generation sequencing of the microbial 16S rRNA genes. A clear difference of the cleanroom microbial community was detected comparing samples from before and during hardware assembly, which was also indicated by a completely different picture of the network and microbial community spreading. The network analysis also revealed that during AIT activities, the cleaning and decontamination procedures reduced the microbial diversity in cleanroom to a very low level, even though the diversity in change room increased drastically, most likely due to high level of utilisation. 
NGS data retrieved from the samples taken during AIT activities showed a lower diversity of microbial community signatures in the cleanrooms, compared to the change room. This is fairly contradictory to the statements made earlier suggesting that cleanrooms carry as diverse microbial communities as the surrounding uncontrolled adjoining facility, based on analyses of a NASA maintained cleanroom [16]. However, it shall be emphasised that the BCCC in Turin was constructed to allow a two-step gowning procedure, where staff changes their own clothes to cleanroom underwear right at the entrance of the complex, and don their cleanroom garment under cleanroom conditions (ISO 7) to reduce the microbial contamination from the change room. This two-step procedure was proposed earlier, since usually the change rooms are the most critical contamination source for cleanrooms [18].

In this study, we used a variety of methods to determine microbiological contamination of the flight hardware and cleanrooms, and performed various alternative cultivation assays, and utilised molecular techniques, including qPCR and next generation sequencing, to assess the quantity and identities of bacterial and archaeal signatures in the cleanrooms. In preparation for the next ESA mission ExoMars 2020, we will use these lessons learned for further optimization of the methodologies. Overall, here we emphasised again the importance of the so-called alternative cultivation assays, for the detection of a broader cultivable diversity, as many bacteria, including several taxa with known resistances, were only detected when a variety of different (special) cultivation media was provided, and the heatshock was not applied prior to cultivation. In addition, the superiority of the NGS-based molecular methods was clearly shown, and with the next mission ExoMars 2020 the cloning and Sanger sequencing method will not be applied any longer, as it will be fully replaced by Illumina MiSeq amplicon sequencing. Notable, the quantitative PCR method did not provide us reasonable information on the absolute microbial abundance during the study, due to the highly sensitive detection of signatures from dead and disrupted microorganisms, whereas the bioburden and alternative assays focus on the cultivable portion of the microbial load. The most informative information was retrieved from NGS analyses, and combination of bioburden and alternative assays: Bioburden assays to determine a proxy for the microbiological contamination of the flight hardware and cleanrooms, and alternative cultivation assays to reveal the cultivable biodiversity of the cleanrooms will be utilised in similar manner also the future.

\section{Conclusion}

To conclude, we would like to highlight the importance of two-stage gowning process and highly effective cleaning and decontamination regime with alternating $\mathrm{pH}$ levels to reduce the bioburden, detected microbial contamination, and microbial diversity during the assembly, integration and testing activities. During this project, the spacecraft hardware never showed microbial contamination levels above the acceptable limit. Consequently, the cleanroom complex at Thales Alenia Space in Turin is an excellent example of how efficient microbiological control for flight hardware under strict planetary protection constraints can be performed in order to ensure mission success of ongoing and upcoming life detection missions.

\section{Additional files}

Additional file 1: Table S1. Cleaning agents in Turin cleanroom. (XLSX $11 \mathrm{~kb})$

Additional file 2: Table S2. Bioburden analysis of cleanroom and spacecraft hardware samples: D: cleanroom wipe samples. The number of colony forming units (CFU) of heat-shock surviving microorganisms is given. CFU total reflects the number of CFU observed on the agar plates. Pour fraction 0.8 refers to the application of a correction factor, since only $80 \%$ of the entire sample was plated. Wipe efficiency 0.2 adds another correction, due to the efficiency of wipe sampling to recover spores from surfaces of about 20\%. (XLSX $24 \mathrm{~kb}$ )

Additional file 3: Table S3. Overview on the air samples taken during the four sampling campaigns and the measured microbial contamination level per $\mathrm{m} \wedge 3$. (XLSX $10 \mathrm{~kb})$

Additional file 4: Table S4. Biodiversity assay ("alternative assays"): Number of colony forming units (CFUs) per $m \wedge$ 2. Numbers have been corrected by pour fraction and wipe efficiency (see Additional file 1: Table S1). (XLSX $12 \mathrm{~kb}$ )

Additional file 5: Table S5. Sampling locations for molecular analyses (wipe samples). (XLSX $9 \mathrm{~kb}$ )

Additional file 6: Table S6. Txt formatted biom table of MiSeq amplicon data. (TXT $102 \mathrm{~kb}$ )

Additional file 7: Table S7. Txt formatted biom table of Sanger sequencing data. (TXT $15 \mathrm{~kb}$ )

Additional file 8: Sequence data analysis pipelines. (DOCX $49 \mathrm{~kb}$ ) Additional file 9: Table S8. Retrieved microbial isolates, the sampling date, location and enrichment strategy ("Fungi" refers to PD agar, see materials and methods). (XLSX $30 \mathrm{~kb}$ )

Additional file 10: Figure S1. Maldi-Tof dendrogram of the obtained isolates, their isolation source and enrichment condition. (TIFF $1200 \mathrm{~kb}$ )

Additional file 11: Table S9. Details on the isolates. (XLSX $20 \mathrm{~kb}$ )

Additional file 12: Figure S2. Riboprint of all Bacillus mycoides isolates. (TIFF 207 kb)

Additional file 13: Figure S3. Boxplots of the results from quantitative PCR, targeting bacterial 165 rRNA genes (Y-axis: Gene copies per m2). Vertical line indicates the minimum and maximum of three replicates. The grey line reflects the median of three replicates, whereas the box indicates the 1st and third quartile. Each sampling campaign is indicated by a different colour. (TIFF 2627 kb)

Additional file 14: Figure S4. PCA plot depicting the relationships of cleanroom and changing room microbial communities (a) and communities before and after the spacecraft hardware was brought in (b) based on predicted function information. (TIFF $8400 \mathrm{~kb}$ )

Additional file 15: Figure S5. Network analysis of samples taken in September 2013. (TIFF 9424 kb)

Additional file 16: Figure S6. Network analysis of samples taken in December 2014. (TIFF 9538 kb) 


\section{Acknowledgements}

We thank the European Space Agency for funding this project under the contract 4000103794/11/NL/EK. We thank Dr. Harald Huber (University Regensburg) for support and discussion. In addition, we thank Thales Alenia Space for providing metadata and support during sampling.

\section{Funding}

European Space Agency funded this project under the contract $4,000,103,794 / 11 / \mathrm{NL} / \mathrm{EK}$

\section{Availability of data and materials}

Sequence data were submitted to the European Nucleotide Archive (ENA) with the study accession number PRJEB15908.

\section{Authors' contributions}

$\mathrm{PR}, \mathrm{RP}, \mathrm{DM}, \mathrm{GK}$ and $\mathrm{CME}$ designed the project. AA, SB and GK collected samples, and $K K, P R, R P, A A, L W$ and $S B$ performed the experiments and measurements. KK, PR, RP and CME analysed and interpreted the data. KK, $P R$, RP, AP and CME prepared the figures. KK, PR, RP and CME wrote the manuscript, and all other authors commented and corrected it. All authors read and approved the final manuscript.

\section{Ethics approval and consent to participate}

Not applicable.

\section{Consent for publication}

Not applicable.

\section{Competing interests}

The authors declare that they have no competing interests.

\section{Publisher's Note}

Springer Nature remains neutral with regard to jurisdictional claims in published maps and institutional affiliations.

\section{Author details}

'Department for Internal Medicine, Section of Infectious Diseases and Tropical Medicine, Medical University of Graz, Graz, Austria.

${ }^{2}$ BioTechMed-Graz, Graz, Austria. ${ }^{3}$ Radiation Biology Department, German Aerospace Center (DLR), Institute of Aerospace Medicine, Cologne, Germany. ${ }^{4}$ Leibniz-Institute DSMZ - German Collection of Microorganisms and Cell Cultures, Braunschweig, Germany. ${ }^{5}$ Department for Microbiology, University of Regensburg, Regensburg, Germany. ${ }^{6}$ Institute of Environmental Biotechnology, Graz University of Technology, Graz, Austria. 'Thales Alenia Space, Turin, Italy. ${ }^{8}$ European Space Agency, Noordwijk, The Netherlands.

\section{Received: 19 April 2017 Accepted: 27 September 2017}

Published online: 25 October 2017

\section{References}

1. Petigura EA, Howard AW, Marcy GW. Prevalence of Earth-size planets orbiting sun-like stars. Proc Natl Acad Sci National Acad Sciences. 2013;110: 19273-8.

2. Rettberg P, Anesio AM, Baker VR, Baross JA, Cady SL, Detsis E, et al. Planetary protection and Mars special regions - a suggestion for updating the definition. Astrobiology. 2016;16(2):119-25.

3. Kminek $G$, Rummel JD. COSPAR's planetary protection policy. Sp Res Today. 2015; Available: https://cosparhq.cnes.fr/sites/default/files/ppp_ article linked to ppp_webpage.pdf

4. Formisano V, Atreya S, Encrenaz T, Ignatiev N, Giuranna M. Detection of methane in the atmosphere of Mars. Science. 2004;306:1758-61.

5. Webster CR, Mahaffy PR, Atreya SK, Flesch GJ, Mischna MA, Meslin P-Y, et al. Mars methane detection and variability at gale crater. Science (80-). 2015: 347:415-7.

6. Kirschke $S$, Bousquet $P$, Ciais $P$, Saunois $M$, Canadell JG, Dlugokencky EJ, et al. Three decades of global methane sources and sinks. Nat Geosci. 2013;6: 813-23.

7. Sender R, Fuchs S, Milo R. Revised estimates for the number of human and bacteria cells in the body. bioRxiv. 2016; doi:10.1101/036103.

8. Meadow JF, Altrichter AE, Bateman AC, Stenson J, Brown GZ, Green لL, et al. Humans differ in their personal microbial cloud. PeerJ. 2015;3:e1258.
9. Mora M, Mahnert A, Koskinen K, Pausan MR, Oberauner-Wappis L, Krause R, et al. Microorganisms in confined habitats: microbial monitoring and control of the International Space Station, cleanrooms, operating rooms and intensive care units. Front Microbiol. 2016;7:1573.

10. Mahnert A, Moissl-Eichinger C, Berg G, Vaishampayan PA, Probst AJ, Auerbach AK, et al. Molecular bacterial community analysis of clean rooms where spacecraft are assembled. Moldenhauer J, editor. Astrobiology. 2016/ 02/03 ed. Pasadena, CA: Elsevier; 2015;3: 1. doi:10.1126/science.aad1329.

11. Moissl-Eichinger C, Cockell C, Rettberg P. Venturing into new realms? Microorganisms in space. FEMS Rev. 2016; doi:10.1093/femsre/fuw015.

12. Puleo JR, Fields ND, Bergstrom SL, Oxborrow GS, Stabekis PD, Koukol R. Microbiological profiles of the Viking spacecraft. Appl Environ Microbiol. 1977:33:379-84.

13. Microbial examination of flight hardware and cleanrooms ECSS-Q-ST-7055C. 2008.

14. Administration NA and S. Handbook for the microbial examination of space hardware: NASA Tech Handb; 2010.

15. Vaishampayan P, Osman S, Andersen G, Venkateswaran K. High-density 165 microarray and clone library-based microbial community composition of the Phoenix spacecraft assembly clean room. Astrobiology. 2010;10 doi:10. 1089/ast.2009.0443.

16. Mahnert A, Vaishampayan P, Probst AJ, Auerbach A, Moissl-Eichinger C, Venkateswaran $\mathrm{K}$, et al. Cleanroom maintenance significantly reduces abundance but not diversity of indoor microbiomes. PLoS One. 2015;10: e0134848.

17. Vaishampayan P, Probst AJ, La Duc MT, Bargoma E, Benardini JN, Andersen $\mathrm{GL}$, et al. New perspectives on viable microbial communities in low-biomass cleanroom environments. ISME J. 2013:7·312-24.

18. Moissl-Eichinger C, Auerbach AK, Probst AJ, Mahnert A, Tom L, Piceno Y, et al. Quo vadis? Microbial profiling revealed strong effects of cleanroom maintenance and routes of contamination in indoor environments. Sci Rep. 2015:5:9156. Available: http://www.nature.com/srep/2015/150317/ srep09156/full/srep09156.html

19. Probst A, Facius R, Wirth R, Moissl-Eichinger C. Validation of a nylon-flockedswab protocol for efficient recovery of bacterial spores from smooth and rough surfaces. Appl Environ Microbiol. 2010;76:5148-58.

20. Vaishampayan P, Probst A, Krishnamurthi S, Ghosh S, Osman S, McDowall A, et al. Bacillus horneckiae sp. nov., isolated from a spacecraft-assembly clean room. Int J Syst Evol Microbiol. 2010;60:1031-7.

21. Behrendt U, Schumann P, Stieglmeier M, Pukall R, Augustin J, Spröer C, et al Characterization of heterotrophic nitrifying bacteria with respiratory ammonification and denitrification activity-description of Paenibacillus uliginis sp. nov., an inhabitant of fen peat soil and Paenibacillus purispatii sp. nov., isolated from a spacec. Syst Appl Microbiol. 2010;33:328-36.

22. Weinmaier T, Probst AJ, Duc MT, Ciobanu D, Cheng J-F, Ivanova N, et al. A viability-linked metagenomic analysis of cleanroom environments: eukarya, prokaryotes, and viruses. Microbiome. 2015:3:1.

23. Moissl-Eichinger C, Pukall R, Probst AJ, Stieglmeier M, Schwendner P, Mora $M$, et al. Lessons learned from the microbial analysis of the Herschel spacecraft during assembly, integration, and test operations. Astrobiology. 2013:13:1125-39. doi:10.1089/ast.2013.1024.

24. Moissl-Eichinger C. Archaea in artificial environments: their presence in global spacecraft clean rooms and impact on planetary protection. ISME J. 2011;5:209-19. Available: http://www.pubmedcentral.nih.gov/articlerender. fcgi?artid $=3105705 \&$ tool $=$ pmcentrez\&rendertype $=$ abstract

25. Moissl-Eichinger $C$, Rettberg P, Pukall R. The first collection of spacecraft-associated microorganisms: a public source for extremotolerant microorganisms from spacecraft assembly clean rooms. Astrobiology. 2012;12:1024-34. Available: http://www.ncbi.nlm.nih.gov/ pubmed/23121015

26. Venkateswaran K, Vaishampayan P, Benardini lii JN, Rooney AP, Spry JA. Deposition of extreme-tolerant bacterial strains isolated during different phases of Phoenix spacecraft assembly in a public culture collection. Astrobiology. 2014;14:24-6.

27. Watve M, Shejval V, Sonawane C, Rahalkar M, Matapurkar A, Shouche Y, Patole M, Phadnis N, Champhenkar A, Damle K, Karandikar S, Kshirsagar V, Jog M. The "K" selected oligophilic bacteria: a key to uncultured diversity? Curr Sci. 2000:78:1535-42.

28. Schumann P, Maier T. MALDI-TOF mass spectrometry applied to classification and identification of bacteria. Methods Microbiol Elsevier. 2014; 41:275-306. 
29. Burggraf S, Olsen GJ, Stetter KO, Woese CR. A phylogenetic analysis of Aquifex pyrophilus. Syst Appl Microbiol Elsevier. 1992;15:352-6.

30. Lane D. 16S/23S rRNA sequencing. In: Stackebrandt E, Goodfellow M, editors. Nucleic acid techniques in bacterial systematics. Chichester: Wiley; 1991. p. 115-75.

31. Caporaso JG, Lauber CL, Walters WA, Berg-Lyons D, Huntley J, Fierer N, et al. Ultra-high-throughput microbial community analysis on the Illumina HiSeq and MiSeq platforms. ISME J. 2012;6:1621-4.

32. Schloss PD, Westcott SL, Ryabin T, Hall JR, Hartmann M, Hollister EB, et al. Introducing mothur: open-source, platform-independent, communitysupported software for describing and comparing microbial communities. Appl Environ Microbiol. 2009;75:7537-41.

33. Quast C, Pruesse E, Yilmaz P, Gerken J, Schweer T, Yarza P, et al. The SILVA ribosomal RNA gene database project: improved data processing and webbased tools. Nucleic Acids Res. 2013:41:D590-6.

34. Hillman-Jackson J, Clements D, Blankenberg D, Taylor J, Nekrutenko A, Team G. Using galaxy to perform large-scale interactive data analyses. Curr Protoc Bioinform. 2012;38:10-5.

35. Shannon P, Markiel A, Ozier O, Baliga NS, Wang JT, Ramage D, et al. Cytoscape: a software environment for integrated models of biomolecular interaction networks. Genome Res Cold Spring Harbor Lab. 2003;13:2498-504.

36. Caporaso JG, Kuczynski J, Stombaugh J, Bittinger K, Bushman FD, Costello EK, et al. QIIME allows analysis of high-throughput community sequencing data. Nat Methods. 2010;7:335-6.

37. McDonald D, Price MN, Goodrich J, Nawrocki EP, Desantis TZ, Probst A. An improved Greengenes taxonomy with explicit ranks for ecological and evolutionary analyses of bacteria and archaea. ISME J. 2012;6 doi:10.1038/ ismej.2011.139.

38. Langille MGI, Zaneveld J, Caporaso JG, McDonald D, Knights D, Reyes JA, et al. Predictive functional profiling of microbial communities using $16 \mathrm{~S}$ rRNA marker gene sequences. Nat Biotechnol. 2013;31:814-21.

39. Moiss-Eichinger C. Extremophiles in spacecraft assembly clean rooms. In: Stan-Lotter H, Fendrihan S. (eds) Adaption of Microbial Life to Environmental Extremes. Vienna: Springer; 2012

40. Segata N, Izard J, Waldron L, Gevers D, Miropolsky L, Garrett WS, et al. Metagenomic biomarker discovery and explanation. Genome Biol. 2011; 12:R60. doi:10.1186/gb-2011-12-6-r60.

41. Branquinho R, Sousa C, Lopes J, Pintado ME, Peixe LV, Osório H. Differentiation of Bacillus Pumilus and bacillus safensis using MALDITOF-MS. PLoS One. 2014;9:e110127.

42. Starostin KV, Demidov EA, Bryanskaya AV, Efimov VM, Rozanov AS, Peltek SE. Identification of bacillus strains by MALDI TOF MS using geometric approach. Sci Rep. 2015:5, 16989

43. Hagen CA, Hawrylewicz EJ, Ehrlich R. Survival of microorganisms in a simulated Martian environment. II. Moisture and oxygen requirements for germination of Bacillus Cereus and Bacillus Subtilis Var. niger spores. Appl Microbiol. 1967;15:285-91.

44. Hawrylewicz E, Gowdy B, Ehrlich R. Micro-organisms under a simulated Martian environment. London: Nature Publishing Group; 1962.

45. Imshenetskiĭ AA, Murzakov BG, Evdokimova MD, Dorofeeva IK. Survival of bacteria in the artificial Mars unit. Mikrobiologiia. 1983;53:731-7.

46. Horneck G, Moeller R, Cadet J, Douki T, Mancinelli RL, Nicholson WL, et al. Resistance of bacterial endospores to outer space for planetary protection purposes-experiment PROTECT of the EXPOSE-E mission. Astrobiology. 2012;12:445-56.

47. Fajardo-Cavazos P, Link L, Melosh HJ, Nicholson WL. Bacillus Subtilis spores on artificial meteorites survive hypervelocity atmospheric entry: implications for lithopanspermia. Astrobiology. 2005;5:726-36.

48. Wassmann M, Moeller R, Rabbow E, Panitz C, Horneck G, Reitz G, et al. Survival of spores of the UV-resistant Bacillus Subtilis strain MW01 after exposure to low-earth orbit and simulated martian conditions: data from the space experiment ADAPT on EXPOSE-E. Astrobiology. 2012;12:498-507.

49. Hotchin J, Lorenz P, Hemenway C. Survival of micro-organisms in space. Nature. 1965;206:442-5.

50. Horneck G, Bücker H, Reitz G. Long-term survival of bacterial spores in space. Adv Sp Res Elsevier. 1994;14:41-5.

51. Brandstätter F, Brack A, Baglioni P, Cockell CS, Demets R, Edwards HGM, et al. Mineralogical alteration of artificial meteorites during atmospheric entry. The STONE-5 experiment. Planet space Sci. Elsevier. 2008;56:976-84.

52. Zhukova Al, Kondratyev II. On artificial Martian conditions reproduced for microbiological research. Life Sci Space Res. 1964;3:120-6.
53. Parfenov GP, Lukin AA. Results and prospects of microbiological studies in outer space. Space Life Sci Springer. 1973:4:160-79.

54. von Wintzingerode F, Rainey FA, Kroppenstedt RM, Stackebrandt E. Identification of environmental strains of bacillus mycoides by fatty acid analysis and species-specific 165 rDNA oligonucleotide probe. FEMS Microbiol Ecol. 1997;24:201-9.

55. Zhang G, Niu F, Busse H-J, Ma X, Liu W, Dong M, et al. Hymenobacter psychrotolerans sp. nov., isolated from the Qinghai-Tibet plateau permafrost region. Int J Syst Evol Microbiol. 2008;58:1215-20.

56. Hirsch P, Ludwig W, Hethke C, Sittig M, Hoffmann B, Gallikowski CA. Hymenobacter roseosalivarius gen. Nov., sp. nov. from continental Antarctic soils and sandstone: bacteria of the Cytophaga/Flavobacterium/Bacteroides line of phylogenetic descent. Syst Appl Microbiol. 1998;21:374-83.

57. Gneiding K, Frodl R, Funke G. Identities of microbacterium spp. encountered in human clinical specimens. J Clin Microbiol. 2008:46:3646-52.

58. Knief C, Ramette A, Frances L, Alonso-Blanco C, Vorholt JA. Site and plant species are important determinants of the methylobacterium community composition in the plant phyllosphere. ISME J. 2010;4:719-28.

59. Paulino-Lima IG, Fujishima K, Navarrete JU, Galante D, Rodrigues F, AzuaBustos $\mathrm{A}$, et al. Extremely high UV-C radiation resistant microorganisms from desert environments with different manganese concentrations. J Photochem Photobiol B Biol. 2016;163:327-36.

60. Bashir M, Ahmed M, Weinmaier T, Ciobanu D, Ivanova N, Pieber TR, et al. Functional Metagenomics of spacecraft assembly cleanrooms: presence of virulence factors associated with human pathogens. Front Microbiol. 2016;7: 1321. doi:10.3389/fmicb.2016.01321.

61. Salter SJ, Cox MJ, Turek EM, Calus ST, Cookson WO, Moffatt MF, Turner P, Parkhill J, Loman NJ, Walker AW. Reagent and laboratory contamination can critically impact sequence-based microbiome analyses. BMC Biol. 2014;12:87. doi:10.1186/s12915-014-0087-z

\section{Submit your next manuscript to BioMed Central and we will help you at every step:}

- We accept pre-submission inquiries

- Our selector tool helps you to find the most relevant journal

- We provide round the clock customer support

- Convenient online submission

- Thorough peer review

- Inclusion in PubMed and all major indexing services

- Maximum visibility for your research

Submit your manuscript at www.biomedcentral.com/submit
) Biomed Central 